\title{
Tricuspid annular plane systolic excursion is significantly reduced during uncomplicated coronary artery bypass surgery: A prospective observational study
}

Andre Korshin, MD, ${ }^{\mathrm{a}}$ Lars Grønlykke, MD, ${ }^{\mathrm{a}}$ Jens Christian Nilsson, MD, PhD, ${ }^{\mathrm{a}}$ Hasse Møller-Sørensen, MD, ${ }^{\mathrm{a}}$ Nikolaj Ihlemann, MD, PhD, ${ }^{\mathrm{b}}$ Sven Morten Kjøller, MD, Sune Damgaard, MD, PhD, ${ }^{c}$ Per Lehnert, MD, PhD,${ }^{c}$ Christian Hassager, MD, DMSc, ${ }^{b}$ Jesper Kjaergaard, MD, PhD, DMSc, ${ }^{b}$ and Hanne Berg Ravn, MD, PhD, DMSc ${ }^{a}$

\section{ABSTRACT}

Objectives: Longitudinal shortening constitutes most of the right ventricle (RV) contraction in the normal heart. However, after even uncomplicated cardiac surgery with preserved RV function a significant and sustained decrease in longitudinal contraction expressed as a reduction in tricuspid annular plane systolic excursion (TAPSE) has been observed. Why and exactly when this happens remains unsettled. The aim of this study was to evaluate the magnitude and timing of changes in TAPSE in relation to sternotomy, pericardial opening, cardiopulmonary bypass $(\mathrm{CPB})$, and chest closure.

Methods: Fifty patients with normal preoperative ejection fraction and no valvulopathy, who underwent coronary artery bypass grafting with the use of CPB, were included. TAPSE was assessed using transthoracic echocardiography (TTE) at baseline and immediately after chest closure. Transesophageal echocardiography was performed at the following time points: after (1) anesthesia induction and transthoracic echocardiography; (2) sternotomy; (3) pericardiotomy; (4) completion of $\mathrm{CPB}$; and (5) chest closure.

Results: TAPSE was significantly reduced to approximately half of its initial value in all patients (from 22 [95\% confidence interval, $21-23 \mathrm{~mm}]$ after anesthesia induction to 9 [95\% confidence interval, $8-10 \mathrm{~mm}$ ] after chest closure). No change was seen after pericardiotomy. The most prominent reduction $(30 \%-40 \%)$ was observed after weaning from CPB. An additional significant decrease of $13 \%$ to $16 \%$ was seen after chest closure.

Conclusions: TAPSE was consistently reduced to approximately half of its initial value after uncomplicated coronary artery bypass grafting surgery. The reduction happened mainly after weaning from $\mathrm{CPB}$, possibly reflecting conformational change of the RV. (J Thorac Cardiovasc Surg 2019;158:480-9)

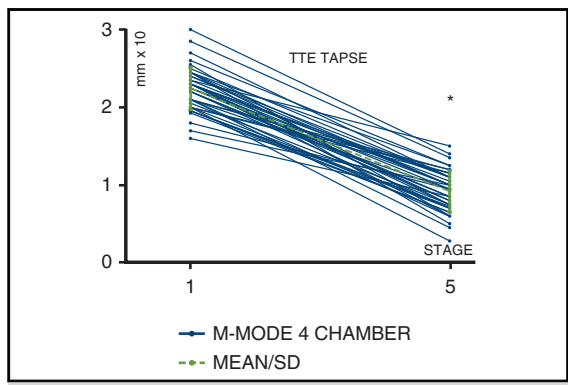

TAPSE reduction by transthoracic echocardiography before and after standard CABG surgery.

\section{Central Message}

After CABG surgery, TAPSE was reduced to approximately half of initial values. This happened after weaning from cardiopulmonary bypass and closure of the chest. It was not related to poor outcomes.

\section{Perspective}

TAPSE reduction observed after weaning from cardiopulmonary bypass possibly reflects conformational change of the right ventricular contraction pattern. The decrease seen after chest closure could be caused by restriction and functional impairment. These findings should be taken into consideration when evaluating right ventricular function following cardiac surgery.

See Commentary on page 490 .

From the Departments of ${ }^{\mathrm{a} C}$ Cardiothoracic Anaesthesiology, ${ }^{\mathrm{b}}$ Cardiology, and ${ }^{\mathrm{c}}$ Cardiothoracic Surgery, The Heart Centre, Rigshospitalet, Denmark.

Received for publication April 19, 2018; revisions received Sept 23, 2018; accepted for publication Sept 27, 2018; available ahead of print Dec 6, 2018.

Address for reprints: Andre Korshin, MD, Rigshospitalet, Department of Cardiothoracic Anesthesiology, Blegdamsvej 7, 4241, 2300 Copenhagen, Denmark (E-mail: akorshin2@gmail.com).

$0022-5223 / \$ 36.00$

Copyright (c) 2018 by The American Association for Thoracic Surgery

https://doi.org/10.1016/j.jtcvs.2018.09.114

Tricuspid annular plane systolic excursion (TAPSE) is regarded as a robust measure of right ventricle (RV) function. ${ }^{1,2}$ Because of previous studies that indicated that RV longitudinal 


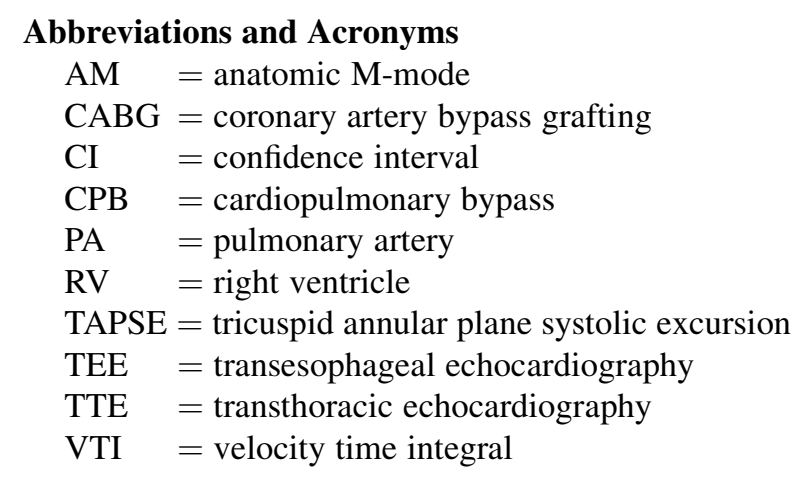

contraction is altered after cardiac surgery, TAPSE for RV assessment in this setting has not been settled. ${ }^{3-5}$ Observations before and after surgery suggest that a reduction in TAPSE occurs without a concomitant decrease in RV output, but rather represents a change in its complex contraction pattern causing more dependency on free wall contraction. $^{6-10}$ Less evidence exists about the RV contraction pattern during ongoing cardiac surgery. The reduction in TAPSE has been reported to occur in association with opening of the pericardial sac. ${ }^{9}$ Unsworth and colleagues observed a reduction in tissue Doppler velocity occurring the moment the pericardium was opened. ${ }^{11}$ However, these observations were discrepant from data reported by Pinto and colleagues, who described that lateral tricuspid annular motion did not change until after weaning from cardiopulmonary bypass $(\mathrm{CPB}){ }^{4}$ This observation, was confirmed more recently by Bitcon and Tousignant. ${ }^{5}$ These conflicting observations might relate to the fact that previous studies comprised small groups of heterogeneous patients and the lack of established validity in the methods used to assess TAPSE perioperatively. Despite the diverging observations on the timing of the reduction in TAPSE, it is assumed not to represent impairment in RV function, but rather an altered overall contraction pattern. ${ }^{1,7,12}$ In the present study, we wanted to evaluate changes in TAPSE in a homogenous group of patients with a low risk of developing RV failure with a validated method of measuring TAPSE perioperatively. More specifically, we wanted to quantitate the magnitude of changes in TAPSE at prespecified time points during a standardized cardiac surgical procedure. A group of patients was chosen that most likely would not exhibit RV failure to elucidate the basic characteristics of the reduction phenomenon. For this purpose, we conducted a prospective observational study in a group of patients with normal heart function, before their coronary artery bypass grafting $(\mathrm{CABG})$ surgery.

\section{METHODS \\ Design}

The study period started January 1, 2015 and ended September 1, 2016. It was a single-center prospective observational cohort study, for which the
Danish Capital Region Local Ethics Committee decided its jurisdiction did not apply, because the analysis qualified as quality assurance (protocol $\mathrm{H}$ 4-2014-FSP). All data handling was in accordance with Danish law and was monitored by the Danish Data Protection Agency (journal 2012-580004 RH-2017-78, I-Suite: 05350) and the Danish Patient Safety Authority.

\section{Patients}

Patients older than the age of 18 years, with an ejection fraction of $>50 \%$, scheduled for elective CABG surgery with the same head surgeon or 1 of his 2 junior faculty surgeons using transesophageal echocardiography (TEE) monitoring were consecutively included in the study. Patients with more than mild grade heart valve pathology, nonsinus rhythm, more than mild grade pulmonary hypertension (estimated RV systolic pressure $>25 \mathrm{~mm} \mathrm{Hg}$ ), or heart malformations diagnosed preoperatively or in the operating room were excluded from the study. The institution's standard practice for the management of patients undergoing CABG surgery was followed. All treatment decisions were left to the treating anesthesiologist and cardiac surgeon. The patient flow is shown in Figure 1.

\section{Procedure}

The institution's standard for CABG surgery consists of general anesthesia with standard American Society of Anesthesiology monitoring as well as invasive vascular access with pressure transducing of the left radial artery and the right internal jugular vein. Anesthesia induction was done using a combination of propofol, midazolam, and fentanyl, and maintained with a combination of sevoflurane, fentanyl, and remifentanil at the choice of the treating anesthesiologist. After anesthesia induction and tracheal intubation, transthoracic echocardiography (TTE) was performed followed by the placement of a TEE probe in the esophagus.

The surgical procedure comprised the following steps: after skin incision the chest was opened using a full median sternotomy and the left internal mammary artery was mobilized for graft usage, after which the mediastinum was incised and full pericardiotomy was attained. The pericardium was suspended and the aorta and the right atrium were cannulated. The right atrium was cannulated through its appendage with a 2-stage venous cannula of appropriate size. CPB was used for all patients. After weaning from $\mathrm{CPB}$, relief of the pericardial suspension and heparin reversal with protamine-sulphate TEE monitoring and image acquisition were continued until skin closure. The pericardial suspension was relieved, but the pericardium was not closed. After chest and skin closure the TEE probe was removed. Another TTE was obtained after the surgical drapes had been removed. Patients were transferred to the cardiac intensive care unit for postoperative management according to the institution's standard for fast-track cardiac surgery. The patient data were assessed at the end of the study period (a minimum of 3 months) for survival outcome via the Danish Civil Registry, which is a unique complete national registry, providing up to date data on survival of all citizens and through the Electronic Health Record Data for other clinical outcomes (discharge time from the Intensive Care Unit, New York Heart Association score at 3month follow-up, inotropic support, acute kidney injury, reoperation from any cause, acute myocardial infarction, heart tamponade, postoperative bleeding of more than $1000 \mathrm{cc}$, wound infection, cerebrovascular events, prolonged ventilatory support, and cardiac arrhythmias).

\section{Echocardiography}

Acquisition of echocardiographic images was divided into the following stages: (1) before chest opening; (2) after chest opening; (3) after full pericardiotomy (but before pericardial suspension); (4) after weaning from CPB (defined as after removal of all cannulas, relief of the pericardial suspension, and complete administration of protamine); and last, (5) after chest closure. Surgery was paused at each time point to accommodate high-quality image acquisition. In stage 1 and 5 TTE was performed in addition to TEE as described previously. Measurements were acquired after 
53 Consecutive patients for elective CABG

with preoperative normal assessment of heart function

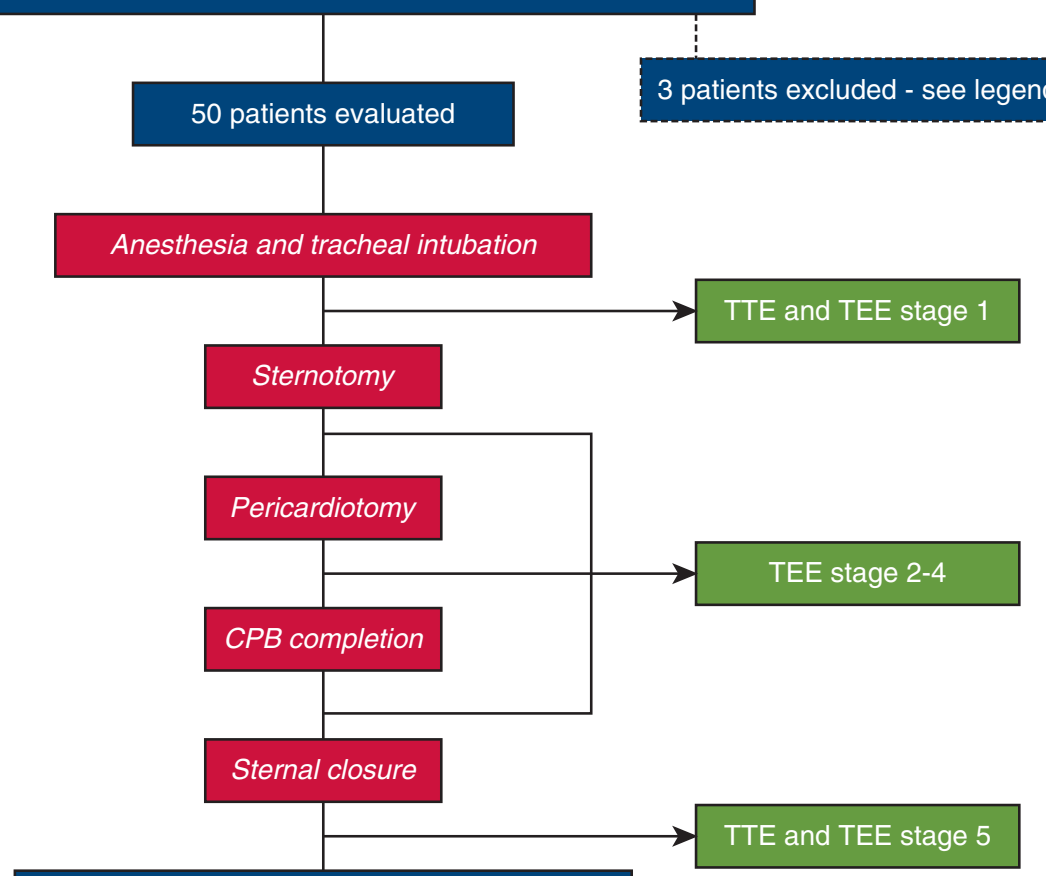

ICU (Fast track management extubation)

Follow-up (minimum 3 months)

FIGURE 1. Patient inclusion and the sequence of transthoracic (TTE) and transesophageal (TEE) echocardiographic exam at the 5 different stages during surgery. Stage 1 is after anesthesia induction, 2 is after sternotomy, 3 is after pericardiotomy, 4 is after weaning from cardiopulmonary bypass $(C P B)$, and stage 5 is after sternal and skin closure. Three patients were excluded; 2 because of atrial fibrillation once in the operating room and 1 because of poor imaging quality. $C A B G$, Coronary artery bypass grafting; $I C U$, intensive care unit.

a period of hemodynamic stability, which was defined as 3 minutes of no more than 10 beats per minute change in heart rate, and $5 \mathrm{~mm} \mathrm{Hg}$ and $3 \mathrm{~mm} \mathrm{Hg}$ variation in mean arterial pressure and central venous pressure, respectively. No vasoactive drugs were administered immediately before the observation period. The TTE at stage 1 was performed after anesthesia induction and after insertion of an endotracheal tube and the TTE at stage 5 was done immediately after surgical drapes had been removed. The TTE and concomitant TEE image acquisition were performed within 10 minutes of each other.

All echocardiographic images were acquired twice 3 to 5 minutes apart over 5 consecutive cardiac cycles synchronized with the patient's electrocardiogram. Image acquisition was achieved in 35 patients using a Philips IE33 machine (Philips Healthcare, Inc, Andover, Mass). In the remaining 15 patients a Philips EPIQ7C machine (Philips Healthcare, Inc) was available. For TTE imaging a S5-1 (1-MHz) probe was used and for TEE imaging an X7-2t (2-MHz) probe was used (Philips Healthcare, Inc). Imaging data were stored for later off-line analysis. TTE TAPSE was imaged using the traditional method with M-mode of the lateral corner of the tricuspid annulus in the apical 4-chamber view in stage 1 and 5 .

TEE TAPSE was acquired in all patients at all 5 stages in the transgastric view with $\mathrm{M}$-mode and anatomic M (AM)-mode. The AM-mode could only be applied in patients scanned with a Philips EPIQ7C machine (Philips Healthcare, Inc), which was available for the last 15 patients in the study. In AM-mode TAPSE is optimized by reorienting the cursor-vector to achieve maximal excursion. This method of measuring TAPSE is featured in Video 1. This will be larger than for conventional M-mode. This technique has been previously validated. ${ }^{13}$ An experienced cardiac anesthesiologist (A.K.), with extensive echocardiographic experience and

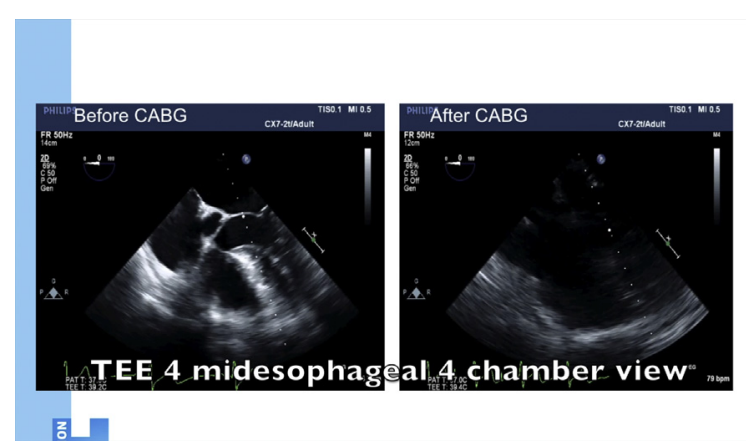

VIDEO 1. Author explaining the main findings in the study. Video available at: https://www.jtcvs.org/article/S0022-5223(18)32782-X/fulltext. 
TABLE 1. Patient characteristics

\begin{tabular}{|c|c|c|}
\hline Characteristic & n or mean & $\begin{array}{l}\text { Percent or } \\
\text { range/SD }\end{array}$ \\
\hline Age, y & 68.8 & $47-84 / 8.7$ \\
\hline Female sex & 9 & 18 \\
\hline Body mass index & 27.4 & $18.9-39.4 / 5.7$ \\
\hline Creatinine, $\mu \mathrm{mol} / \mathrm{L}$ & 92 & $50-198 / 28.1$ \\
\hline Left ventricular ejection fraction & 0.55 & $0.45-0.60 / 5.3$ \\
\hline Non-coronary heart pathology & $\begin{array}{c}3 \text { (mild aortic } \\
\text { stenosis) }\end{array}$ & 6 \\
\hline Diabetes & 14 & 28 \\
\hline Hypertension & 37 & 74 \\
\hline Hyperlipidemia & 42 & 84 \\
\hline $\begin{array}{l}\text { Chronic obstructive pulmonary } \\
\text { disease }\end{array}$ & 7 & 14 \\
\hline Previous stroke & 7 & 14 \\
\hline Previous myocardial infarction & 16 & 32 \\
\hline Right coronary artery disease & 31 & 62 \\
\hline Left dominant coronary anatomy & 6 & 12 \\
\hline $\begin{array}{l}\text { Cardiopulmonary bypass time, } \\
\text { minutes }\end{array}$ & 75.1 & $30-131 / 19.6$ \\
\hline LIMA-LAD & 48 & 96 \\
\hline VG-OM & 33 & 66 \\
\hline VG-PDA & 21 & 42 \\
\hline VG-Dia & 9 & 18 \\
\hline VG-PLA & 6 & 12 \\
\hline VG-IM & 5 & 10 \\
\hline VG-Cx & 4 & 8 \\
\hline VG-RCA & 4 & 8 \\
\hline Rad-Dia & 1 & 2 \\
\hline Rad-OM & 1 & 2 \\
\hline
\end{tabular}

$S D$, Standard deviation; LIMA, left internal mammary artery; $L A D$, left anterior descending; $V G$, vein graft; $O M$, obtuse marginal; $P D A$, posterior descending artery; Dia, diagonal; PLA, posterior left artery; $I M$, intermediate marginal; $C x$, circumflex; $R C A$, right coronary artery; $R a d$, radial.

certified diploma with the National Board of Echocardiography, acquired and read all images.

To assess other contractile indices of RV function we also analyzed tissue Doppler velocity measurements of the lateral tricuspid annulus and pulmonary artery (PA) velocity time at all 5 stages of surgery in all patients. The tissue Doppler velocity was measured in the modified transgastric RV-inflow view. The PA velocity time integral (VTI) was assessed in the upper esophageal ascending aortic short axis view with the pulsed-wave sample volume set at $1 \mathrm{~cm}$ with care taken to place the sample volume the same distance from the probe in the main PA (approximately $4-5 \mathrm{~cm}$ from the esophagus). These echocardiographic techniques have also been previously validates. ${ }^{13}$ All measurements were read off-line as the average of 2 repeated acquisitions. Mean arterial pressures, central venous pressures, and heart rates were recorded at all time points.

All echocardiographic measures were repeated twice separated by 2 to 3 minutes in all 5 stages of surgery. Apneic measurements were not performed. Routine institution policy of 6 to $10 \mathrm{~mL} / \mathrm{kg}$ tidal volume ventilation was used.
TABLE 2. Patient outcome and complications of surgery $(\mathbf{N}=\mathbf{5 0}$ patients)

\begin{tabular}{lrr}
\hline \multicolumn{1}{c}{ Outcome or complication } & n & $\%$ \\
\hline Death at follow-up & 2 & 4 \\
Discharge from ICU within $24 \mathrm{~h}$ & 47 & 94 \\
\hline NYHA score $>3$ at follow-up & 3 & 6 \\
Inotropic support $>24 \mathrm{~h}$ & 1 & 2 \\
AKI (creatinine $\times 2,<0.5 \mathrm{~mL} / \mathrm{kg} / \mathrm{h}$ ) & 1 & 2 \\
Reoperation from any cause at $3 \mathrm{mo}$ & 3 & 6 \\
Acute myocardial infarction & 0 & 0 \\
$\quad$ (clinically evaluated) & &
\end{tabular}

$\begin{array}{lll}\text { Tamponade } & 2 & 4 \\ \text { Bleeding }>1000 \mathrm{~mL} & 3 & 6 \\ \text { Infection } & 0 & 0 \\ \begin{array}{l}\text { Cerebrovascular event (clinically } \\ \text { evaluated) }\end{array} & 0 & 0\end{array}$

Prolonged ventilator support $\quad 1 \quad 2$

Atrial fibrillation $\quad 18 \quad 36$

$\overline{I C U}$, Intensive care unit; NYHA, New York Heart Association; $A K I$, acute kidney injury.

\section{Statistics}

Sample size was calculated using G*power 3.1 (Erdfelder, Faul, \& Buchner, 1996) —analysis of variance 5 repeated measures, within factors-on the basis of previous studies of TAPSE values in cardiac and normal patient populations ${ }^{14}$ to comprise 43 patients with a power of $80 \%(\beta=0.2)$ and significance level of 0.025 to detect a $20 \%$ reduction in TAPSE with a correlation among repeated measures of $0.42 .{ }^{15}$ To account for a presumed availability of $85 \%$ of images we included 50 patients.

Continuous variables are described with mean, range, and 95\% confidence interval (CI), or standard deviation as appropriate. Categorical variables are presented as absolute numbers and proportions. All echocardiographic measures during the 5 stages were subjected to analysis of variance for repeated measures comparing main effects with a CI of $95 \%$ adjusted according to the Bonferroni principle. Comparisons between patient groups was done using independent sample $t$ test. Analyses were performed using SPSS, version 23.0 for Mac (IBM Corp, Armonk, NY) and Prism version 5.0 for Mac OS X (GraphPad Software, La Jolla, Calif) for graph construction.

\section{RESULTS}

\section{Clinical Characteristics and Outcome}

Fifty patients were included in the study. Patient characteristics and outcomes are shown in Tables 1 and 2, respectively. The patients included had few comorbidities, a low rate of complications postoperatively, and short discharge times from the Intensive Care Unit. The gender distribution was skewed toward more male than female patients. There was no significant difference in TAPSE between patients with $(\mathrm{n}=31)$ and without $(\mathrm{n}=19)$ right coronary artery disease before or after surgery $(P=.53$, $P=.37)$. Correlation of pacing, time on $\mathrm{CPB}$, and presence of right coronary artery disease to TAPSE change is shown in Table 3. 
TABLE 3. Comparison of different groups of patients and tricuspid annulus plan systolic excursion before and after surgery

\begin{tabular}{|c|c|c|c|c|c|}
\hline & $\mathbf{n}$ & TAPSE before & $P$ value & TAPSE after & $P$ value \\
\hline \multicolumn{6}{|l|}{ CPB } \\
\hline$\leq 60 \mathrm{~min}$ & 12 & $22.0(3.6)$ & .97 & $8.6(3.0)$ & .43 \\
\hline$>60 \min$ & 36 & $21.9(3.4)$ & & $9.3(2.6)$ & \\
\hline \multicolumn{6}{|l|}{ RCA disease } \\
\hline Present & 31 & $21.8(3.5)$ & .53 & 8.9 (2.9) & .37 \\
\hline Not present & 19 & $22.4(3.2)$ & & $9.6(2.3)$ & \\
\hline \multicolumn{6}{|l|}{$\mathrm{RCA}$} \\
\hline Grafted & 21 & $18.3(3.7)$ & .05 & $9.5(0.8)$ & .84 \\
\hline Not grafted & 29 & $22.3(3.3)$ & & $9.2(2.7)$ & \\
\hline \multicolumn{6}{|c|}{ Rhythm off CPB } \\
\hline Paced & 19 & $21.8(4.0)$ & .73 & $9.3(2.7)$ & .71 \\
\hline Sinus & 31 & $22.2(3.1)$ & & $9.0(2.6)$ & \\
\hline \multicolumn{6}{|c|}{ Pressor/inotropes } \\
\hline Yes & 6 & 22.9 (1.6) & .59 & $10.4(1.9)$ & .36 \\
\hline No & 44 & $22.0(3.5)$ & & $9.1(2.7)$ & \\
\hline
\end{tabular}

TAPSE, Tricuspid annular plane systolic excursion; $C P B$, cardiopulmonary bypass; $R C A$, right coronary artery.

\section{Changes in Echocardiographic Measures During Surgery}

TTE TAPSE decreased significantly in all patients with a mean reduction from the baseline of $22 \mathrm{~mm}(95 \% \mathrm{CI}$, 21-23 mm) after anesthesia induction to $9(95 \% \mathrm{CI}, 8$ $10 \mathrm{~mm}$ ) after chest closure. Repetitive TEE measurements of TAPSE showed a significant reduction after weaning from CPB to $11 \mathrm{~mm}(95 \% \mathrm{CI}, 10-12)$ for $\mathrm{M}$ mode $(P<.001)$ and to $12 \mathrm{~mm}(95 \% \mathrm{CI}, 11-13)$ for AM-mode TAPSE $(P<.001)$. In addition, a significant decrease in TAPSE was seen after chest closure to $9 \mathrm{~mm}(95 \% \mathrm{CI}, 8-9 \mathrm{~mm})$ for M-mode $(P<.01)$ and to
$9 \mathrm{~mm}(95 \% \mathrm{CI}, 8-11 \mathrm{~mm})$ for AM-mode $(P<.01)$. In contrast, there was no significant change in TAPSE after sternotomy or pericardiotomy, compared with baseline (Figure 2). Mean TAPSE values at the individual time points during surgery are shown in Table 4. Tissue Doppler velocity measurements of the lateral tricuspid annulus during surgery revealed a small, but significant reduction after the chest had been closed (stage 5), but otherwise tissue Doppler velocities remained stable without any significant change throughout surgery (Figure 3). A characteristic course of the TAPSE images during surgery is shown in Figure 4.
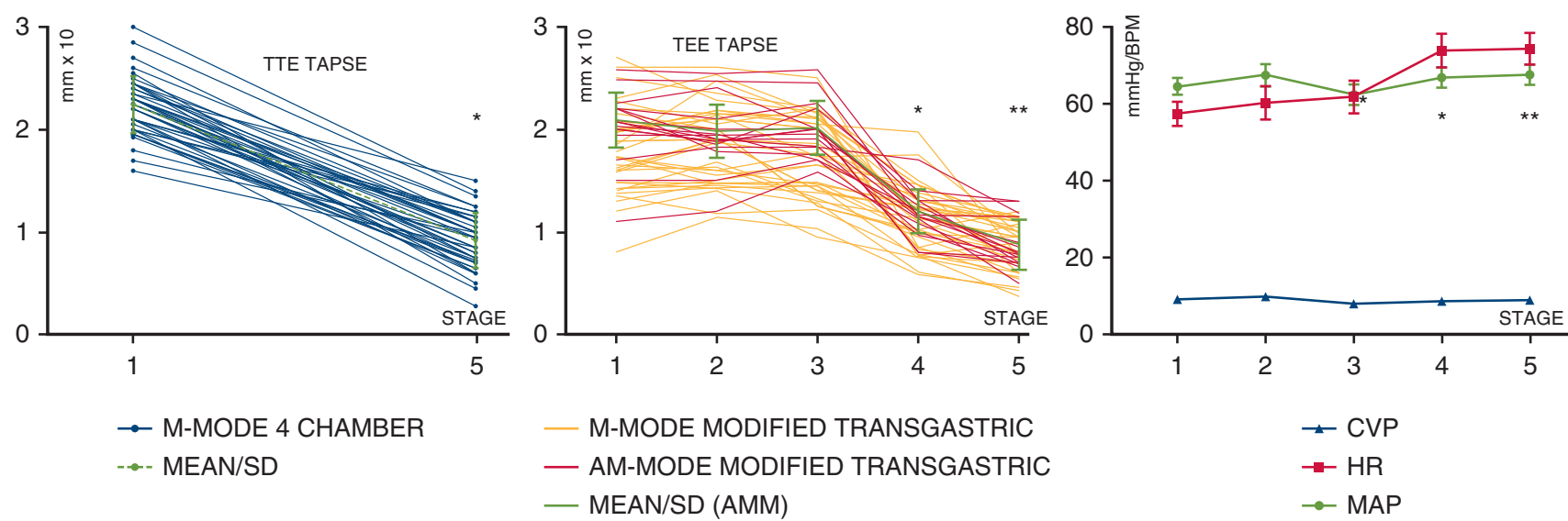

FIGURE 2. Measurements of central venous pressure (CVP), mean arterial pressure $(M A P)$ and heart rate $(H R)$ in 50 patients during 5 predefined stages of surgery. Stage 1: after anesthesia induction, 2: after sternotomy, 3: after pericardiotomy, 4: after weaning from cardiopulmonary bypass, and stage 5: after sternal and skin closure. In addition, echocardiographic measurements of tricuspid annular plane systolic excursion (TAPSE) using transthoracic echocardiography (TTE) in the apical view ( $\mathrm{n}=43$ at stage 1 and 5) and transesophageal echocardiography (TEE) in the transgastric modified right ventricular inflow-outflow view in anatomic M-mode $(A M M ; \mathrm{n}=15,14,14,14$, and 15 , at stage 1-5, respectively) and M-mode ( $\mathrm{n}=34,34,35,35$, and 35, at stage $1-5$, respectively). $* P<.05$ between stage 3 and 4 (for TTE TAPSE between stage 1 and 5 ). $* * P<.05$ between stage 4 and $5 . B P M$, Beats per minute. 
TABLE 4. Hemodynamic changes during the 5 stages of surgery

\begin{tabular}{|c|c|c|c|c|c|}
\hline & $\begin{array}{l}\text { Stage 1: before } \\
\text { skin incision }\end{array}$ & $\begin{array}{l}\text { Stage 2: after } \\
\text { sternotomy }\end{array}$ & $\begin{array}{l}\text { Stage 3: after } \\
\text { pericardiotomy }\end{array}$ & $\begin{array}{l}\text { Stage 4: after } \\
\text { CPB weaning }\end{array}$ & $\begin{array}{l}\text { Stage 5: after } \\
\text { chest closure }\end{array}$ \\
\hline TTE TAPSE $(\mathrm{n}=43,43)$ & $22(21-23) \mathrm{mm}$ & - & - & - & $9(8-10) \mathrm{mm}^{*}$ \\
\hline $\begin{array}{l}\text { TEE TAPSE mmTG } \\
\qquad(\mathrm{n}=49,48,49,49,49)\end{array}$ & $17(15-18) \mathrm{mm}$ & $17(16-18) \mathrm{mm}$ & $16(15-18) \mathrm{mm}$ & $11(10-12) \mathrm{mm}^{*}$ & $9(8-9) \mathrm{mm}^{*}$ \\
\hline $\begin{array}{l}\text { TEE TAPSE ammTG } \\
\quad(\mathrm{n}=15,14,14,14,15)\end{array}$ & $21(19-22)$ & $20(18-21) \mathrm{mm}$ & $20(19-21) \mathrm{mm}$ & $12(11-13) \mathrm{mm}^{*}$ & $9(8-11) \mathrm{mm}^{*}$ \\
\hline $\begin{array}{l}\text { TGTDI }(\mathrm{n}=45,45,46,47 \text {, } \\
\text { 47) }\end{array}$ & $8.1(7.6-8.5) \mathrm{cm} / \mathrm{s}$ & $8.6(8.1-9.2) \mathrm{cm} / \mathrm{s}$ & $10.0(9.2-10.7) \mathrm{cm} / \mathrm{s}$ & $8.1(7.3-9.0) \mathrm{cm} / \mathrm{s}$ & $7.0(6.3-7.6) \mathrm{cm} / \mathrm{s}^{*}$ \\
\hline PAVTI $(\mathrm{n}=50,49,49,49,50)$ & $15.5(14.6-16.4) \mathrm{cm}$ & $14.8(13.8-15.7) \mathrm{cm}$ & $16.1(15.2-17.0) \mathrm{cm}$ & $19.4(18.2-20.6) \mathrm{cm}^{*}$ & $17.0(15.7-18.1) \mathrm{cm}^{*}$ \\
\hline $\mathrm{MAP}(\mathrm{n}=50,49,49,49,50)$ & $64(62-66) \mathrm{mm} \mathrm{Hg}$ & $68(65-70) \mathrm{mm} \mathrm{Hg}$ & $63(60-65) \mathrm{mm} \mathrm{Hg}$ & 67 (65-70) $\mathrm{mm} \mathrm{Hg}$ & $68(65-70) \mathrm{mm} \mathrm{Hg}$ \\
\hline $\operatorname{CVP}(\mathrm{n}=50,50,50,50,50)$ & $9(8-10) \mathrm{mm} \mathrm{Hg}$ & $10(9-11) \mathrm{mm} \mathrm{Hg}$ & $8(7-9) \mathrm{mm} \mathrm{Hg}$ & $9(8-10) \mathrm{mm} \mathrm{Hg}$ & $9(8-10) \mathrm{mm} \mathrm{Hg}$ \\
\hline $\mathrm{HR}(\mathrm{n}=50,50,50,50,50)$ & $58(55-60) \mathrm{bpm}$ & $61(57-65) \mathrm{bpm}$ & $63(59-67) \mathrm{bpm}$ & $74(70-78) b^{2} *$ & 74 (70-78) bpm \\
\hline
\end{tabular}

Data are presented as mean $(95 \%$ CI). MAP, primary $P$ value $<.001 ;$ CVP, primary $P$ value $=.144$; and HR, primary $P$ value $<.001$ as well as TAPSE using TTE and TEE mmTG (primary $P$ value $<.001$ ) and ammTG (primary $P$ value $<.001$ ) views; pulse wave Doppler velocity of the tricuspid annulus with TGTDI (primary $P$ value $<.001$ ) and of the velocity time integral of the PAVTI (primary $P$ value $<.001$ ). $C P B$, Cardiopulmonary bypass; TTE, transthoracic echocardiography; TAPSE, tricuspid annular plane systolic excursion; TEE, transesophageal echocardiography; $m m T G$, in M-mode transgastric; ammTG, anatomic M-mode transgastric; TGTDI, tissue Doppler applied in the modified transgastric view; $P A V T I$, pulmonary artery velocity time integral; $M A P$, mean arterial pressure; $C V P$, central venous pressure; $H R$, heart rate; $b p m$, beats per minute. $* P<.05$ compared with previous stage.

The PA VTI increased by $23 \%(P<.001)$ after weaning from $\mathrm{CPB}$, followed by a decrease of $12 \%$ after sternal closure $(P<.001$; Table 4 and Figure 3). Forty-five patients $(90 \%)$ were weaned from CPB without the use of vasopressor or inotropic support; the remaining 5 required an infusion of low-dose norepinephrine $(<5 \mathrm{ng} / \mathrm{kg} / \mathrm{min})$. One received inotrope infusion.

\section{Hemodynamics During Surgery}

Small fluctuations in central venous pressure, mean arterial pressure, and heart rate were seen during surgery (Table 4). None were significant until after weaning off $\mathrm{CPB}$, when heart rate and mean arterial pressure showed small significant increases $(P<.001, P<.025)$. Most patients $(\mathrm{n}=31 ; 62 \%)$, were weaned from CPB in sinus rhythm (Table 3). Nineteen patients $(38 \%)$ required epicardial pacing; among these, $5(10 \%)$ needed atrial-ventricular pacing and $14(28 \%)$ were atrial paced only. There were no significant differences in TAPSE measurements between patients in sinus rhythm and patients requiring pacing $(P=.71)$.

\section{DISCUSSION}

TAPSE was significantly reduced in all patients after CABG surgery (see Central Image). The most pronounced reduction occurred after weaning from $\mathrm{CPB}$ with an additional reduction occurring at chest closure. These findings were observed in a homogeneous low-risk on-pump CABG patient population with normal initial TAPSE values and an uneventful clinical course postoperatively. The implication of these findings is that TAPSE reduction to half of initial values could be perceived as a "typical"
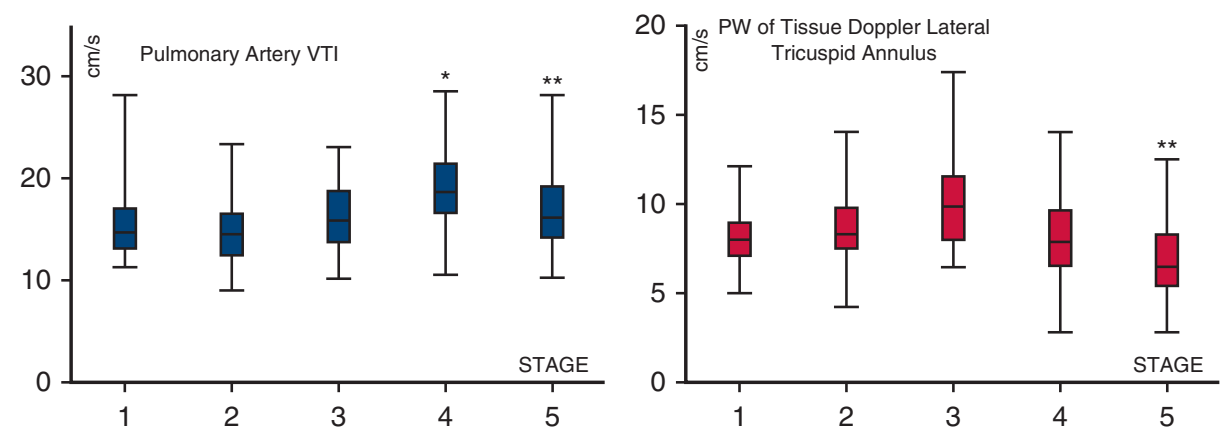

FIGURE 3. Transesophageal echocardiographic measurements of pulmonary artery velocity time integral (VTI) in the upper esophageal ascending aortic short axis view (pulmonary artery VTI $\mathrm{n}=50,49,49,49$, and 50, at stage 1-5, respectively) and tissue Doppler velocity in the transgastric modified right ventricular inflow-outflow view (TGTDI) at stage 1 through 5 (TGTDI $\mathrm{n}=45,45,46,47,47$, at stage 1-5, respectively). Stage 1 is after anesthesia induction, 2 after sternotomy, 3 after pericardiotomy, 4 after weaning from the cardiopulmonary bypass machine, and stage 5 is after sternal and skin closure. $* P<.05$ between stage 3 and 4 . $* * P<.05$ between stage 4 and 5. $P W$, Pulsed wave. 

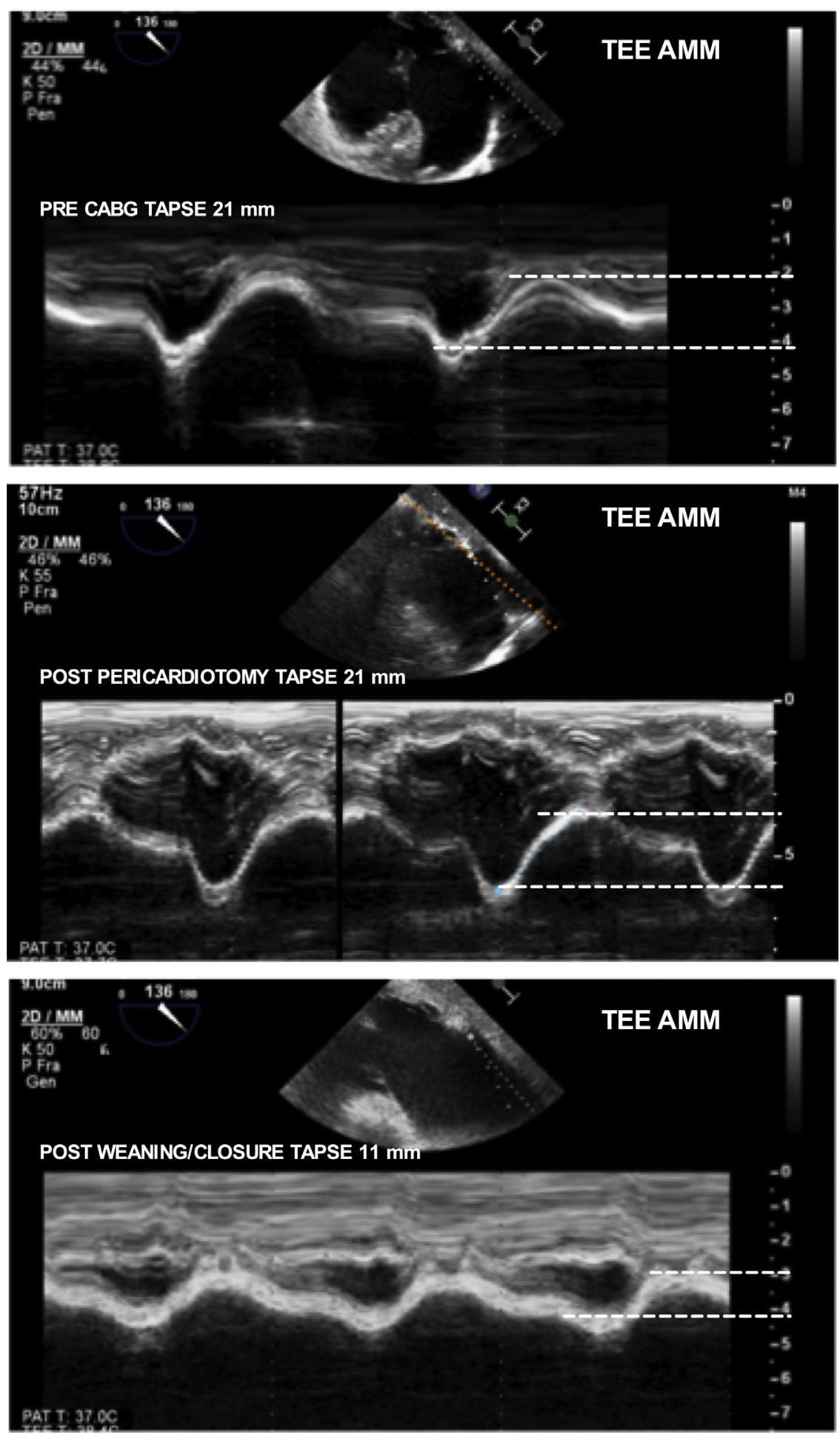

FIGURE 4. Still images of transesophageal echocardiography in anatomic M-mode (TEE AMM) from the transgastric modified right ventricular inflowoutflow view showing the change in tricuspid annular systolic plane excursion (TAPSE) from before surgery (stage 1), after pericardiotomy (stage 3), and at the end of surgery (stage 5). $C A B G$, Coronary artery bypass grafting.

finding in patients who undergo a standard cardiac surgical procedure and not as an indication of impaired RV function.
The RV contraction pattern consistently changed after cardiac surgery. ${ }^{10,16-20}$ Such contraction pattern changes have led to the assumption that TAPSE has limited ability 
to provide meaningful assessment during cardiac surgery and postoperatively in the critical care unit. ${ }^{9,10,15,21-23}$ It is not interventions on the heart in itself causing reduction, because patients with percutaneously replaced aortic valves have been shown to preserve TAPSE values as opposed to patients receiving surgical replacement in a large prospective randomized trial. ${ }^{15,24,25}$ In addition to aortic valve replacement, TAPSE reduction has been shown to occur after CABG,${ }^{16}$ atrial septal and mitral valve repair, ${ }^{10,15,18,19}$ and thus seem to be consistently present after all types of cardiac surgery. Even after procedures in which preservation of TAPSE would be expected like pulmonary endarterectomy it seems to be reduced. ${ }^{26}$ The reduction has been shown to remain postoperatively after 12 months ${ }^{15}$ using echocardiography and even after 32 months using magnetic resonance imaging. ${ }^{18}$ The reduction most likely reflect a change in the contraction pattern, not in RV output, because other RV function parameters have not shown a corresponding reduction. ${ }^{10,16,18}$ Consistent with this hypothesis, an increase in mitral annular plane systolic excursion has been reported, ${ }^{15}$ implying compensation for right heart changes by the left heart, suggesting an altered contraction pattern of the whole heart. ${ }^{15}$

To identify at what point TAPSE change can be expected to occur during surgery and what extent of reduction to anticipate, we conducted a prospective study with timespecified observations. We used TEE, because the open chest cavity throughout surgery hinders evaluation using TTE. A statistical significant reduction in TAPSE was observed after weaning from CPB with an additional smaller reduction occurring after chest closure. Our findings contribute to accumulating evidence ${ }^{3-5}$ suggesting that the time point of major change in the RV contraction pattern occurs after $\mathrm{CPB}$ - not after pericardiotomy. A recent study also investigating TEE TAPSE during surgery likewise reported the reduction in TAPSE to occur after weaning from CPB..$^{5} \mathrm{~A}$ less pronounced reduction was seen in that study, which might be related to the use of a different TEE view (the 4-chamber view) and method for TAPSE assessment (speckle tracking). ${ }^{5}$ The conflicting results described previously by Unsworth and colleagues ${ }^{11}$ when the major reduction was observed after pericardiotomy might be explained by a limited number of observations-only 7 patients were evaluated - and by the method of measurement. In that study the longitudinal contraction was assessed using tissue Doppler velocity in the 4-chamber view-not using TAPSE. We have previously shown that tissue Doppler velocity of the tricuspid annulus in the 4-chamber view ${ }^{11}$ shows a low reproducibility, which might have skewed results.

The access to the heart also influences the reduction in TAPSE; a median sternotomy has been shown to cause a more pronounced reduction than a lateral mini- thoracotomy. ${ }^{16}$ In addition, some degree of pericardial incision has been shown to be required for the reduction to develop. ${ }^{16}$ Considering that some form of chest opening has to be combined with some degree of pericardiotomy for TAPSE reduction to develop and that most results ${ }^{3-5}$ indicate that no changes occur at the time point of pericardiotomy, but rather occurs after $\mathrm{CPB}-2$ mechanisms could be at play-one caused by the combination of pericardiotomy with chest closure-and one occurring during $\mathrm{CPB}$. Because the reduction in TAPSE has also been shown to occur after off-pump CABG,${ }^{17}$ it might not be the CPB in itself causing it. Our results support 2 mechanisms because we observed 2 separate significant reductions-one related to $\mathrm{CPB}$ and one related to chest closure.

In our study RV indices of contractility (PAVTI and tissue Doppler velocity of the tricuspid annulus in the transgastric view) were increased after $\mathrm{CPB}$, also in patients not receiving inotropes, supporting that the reduction in TAPSE does not represent a decrease in RV output, but rather represent a change in contraction pattern. Loading conditions when weaning from CPB could explain increased contractility by recruitment of nonlongitudinal RV contraction. In contrast, chest closure was associated with reduced RV contractile indices. The combination of pericardiotomy and chest closure might cause compression of the heart and friction from the chest wall, adding to the reduction in TAPSE by actually impairing RV output. Chest wall contact impairing RV motion has been observed using magnetic resonance imaging after cardiac surgery. ${ }^{18}$ This might be evident even when the pericardium is surgically restored, because closing the pericardium has been shown not to alleviate the reduction in RV longitudinal contraction. ${ }^{19}$

Because a reduction in TAPSE to at least half of normal values seems to be a typical feature of uncomplicated cardiac surgery, ${ }^{27}$ estimations of RV function after CPB using TAPSE might be problematic. ${ }^{28}$ However, we observed that TAPSE correlated with other RV indices of contractility when the reduction after CPB had occurred. This is in agreement with observations made by Garcia Gigorro and colleagues, ${ }^{29}$ who reported good TAPSE correlation with RV function measured using continuous PA catheter in post cardiac surgery patients. TAPSE as a tool in the assessment of RV function after cardiac surgery thus potentially might still be of value when attention is paid to the typical reduction seen after CPB. However, the only firm conclusion that can be made from the current study is that a TAPSE reduction to approximately half of normal value is a typical finding after cardiac surgery performed with pericardiotomy and sternotomy and does not necessarily indicate RV function impairment.

Exactly what causes the main change during $\mathrm{CPB}$ and how the RV retains output remains to be elucidated. Incorporating 3-D imaging of the RV with speckle tracking 
techniques could evaluate circumferential, radial, and longitudinal contraction of the entire RV at the same point in time and possibly further clarify mechanisms of change in RV contraction pattern. TEE incorporating 3-D technology has been shown to have high feasibility in the setting of cardiac surgery. ${ }^{6}$

Validation and comparison of TEE TAPSE with TTE TAPSE has only been carried out to a limited extent. ${ }^{30,31}$ A strength in the current study was that we applied TEE views and assessment of TAPSE on the basis of observations from a previous investigation..$^{13}$ In that study we found that anatomic M-mode in the transgastric view showed the best performance compared with TTE throughout surgery. Flo Forner and colleagues ${ }^{30}$ have also shown good agreement between TTE and TEE anatomic M-mode TAPSE, but only in the preoperative setting-not throughout surgery. In contrast, the M-mode in the transgastric view although showing high feasibility and reproducibility carries a systematic underestimation of TAPSE compared with TTE. ${ }^{13,30}$ Still, the sequential changes in M-mode TAPSE seen in the current study was in agreement with the anatomic M-mode in the transgastric view, which as described has shown high feasibility, high reproducibility, as well as high interchangeability with TTE TAPSE, thus strengthening the findings.

Limitations apply to our study: it was a single-center study, where the same operator performed all echocardiographic images. We would have preferred to use other indices of RV function, like a PA catheter, but this is not part of the standard care for uncomplicated CABG surgery within our institution. Finally, because of the low-risk surgery the study was not powered to correlate changes in TAPSE with patient outcome.

\section{CONCLUSIONS}

In summary, TAPSE was consistently and significantly reduced to approximately half its initial value in all patients during open-chest surgery using CPB after uncomplicated CABG surgery. Most of the reduction was observed after weaning from $\mathrm{CPB}$, possibly reflecting conformational changes of the RV. A smaller decrease was also seen after chest closure, possibly caused by functional change related to chest restrictions. These findings should be taken into consideration when evaluating the RV function postoperatively in patients during and after cardiac surgery.

\section{Conflict of Interest Statement}

Authors have nothing to disclose with regard to commercial support.

\footnotetext{
References

1. Mitoff PR, Beauchesne L, Dick AJ, Chow BJ, Beanlands RS, Haddad H, et al. Imaging the failing right ventricle. Curr Opin Cardiol. 2012;27:148-53.
}

2. Haddad F, Hunt SA, Rosenthal DN, Murphy DJ. Right ventricular function in cardiovascular disease, part i: anatomy, physiology, aging, and functional assessment of the right ventricle. Circulation. 2008;117:1436-48.

3. Wranne B, Pinto FJ, Hammarström E, St Goar FG, Puryear J, Popp RL. Abnormal right heart filling after cardiac surgery: time course and mechanisms. Br Heart J. 1991;66:435-42.

4. Pinto FJ, Wranne B, St Goar FG, Siegel LC, Haddow G, Schnittger I, et al. Systemic venous flow during cardiac surgery examined by intraoperative transesophageal echocardiography. Am J Cardiol. 1992;69:387-93.

5. Bitcon CJ, Tousignant $\mathrm{C}$. The effect of pericardial incision on right ventricular systolic function: a prospective observational study. Can J Anaesth. 2017;64: 1194-201.

6. Fusini L, Tamborini G, Gripari P, Maffessanti F, Mazzanti V, Muratori M, et al. Feasibility of intraoperative three-dimensional transesophageal echocardiography in the evaluation of right ventricular volumes and function in patients undergoing cardiac surgery. J Am Soc Echocardiogr. 2011;24:868-77.

7. Raina A, Vaidya A, Gertz ZM, Chambers S, Forfia PR. Marked changes in right ventricular contractile pattern after cardiothoracic surgery: implications for postsurgical assessment of right ventricular function. J Heart Lung Transplant. 2013; 32:777-83.

8. Alam M, Hedman A, Nordlander R, Samad B. Right ventricular function before and after an uncomplicated coronary artery bypass graft as assessed by pulsed wave Doppler tissue imaging of the tricuspid annulus. Am Heart J. 2003;146: 520-6.

9. Rösner A, Avenarius D, Malm S, Iqbal A, Schirmer H, Bijnens B, et al. Changes in right ventricular shape and deformation following coronary artery bypass surgery-insights from echocardiography with strain rate and magnetic resonance imaging. Echocardiography. 2015;32:1809-20.

10. Tamborini G, Muratori M, Brusoni D, Celeste F, Maffessanti F, Caiani EG, et al. Is right ventricular systolic function reduced after cardiac surgery? A two- and three-dimensional echocardiographic study. Eur J Echocardiogr. 2009; 10:630-4.

11. Unsworth B, Casula RP, Kyriacou A, Yadav H, Chukwuemeka A, Ashok C, et al. The right ventricular annular velocity reduction caused by coronary artery bypass graft surgery occurs at the moment of pericardial incision. Am Heart J. 2010;159: 314-22.

12. Greyson CR. Right heart failure in the intensive care unit. Curr Opin Crit Care. 2012;18:424-31.

13. Korshin A, Grønlykke L, Nilsson JC, Møller-Sørensen H, Thlemann N, Kjøller M, et al. The feasibility of tricuspid annular plane systolic excursion performed by transesophageal echocardiography throughout heart surgery and its interchangeability with transthoracic echocardiography. Int J Cardiovasc Imaging. 2018;34:1017-28.

14. Tamborini G, Pepi M, Galli CA, Maltagliati A, Celeste F, Muratori M, et al. Feasibility and accuracy of a routine echocardiographic assessment of right ventricular function. Int J Cardiol. 2007;115:86-9.

15. Grønlykke L, Ihlemann N, Ngo AT, Thyregod HG, Kjaergaard J, Korshin A, et al. Measures of right ventricular function after transcatheter versus surgical aortic valve replacement. Interact Cardiovasc Thorac Surg. 2017;24:181-7.

16. Unsworth B, Casula RP, Yadav H, Baruah R, Hughes AD, Mayet J, et al. Contrasting effect of different cardiothoracic operations on echocardiographic right ventricular long axis velocities, and implications for interpretation of postoperative values. Int J Cardiol. 2013;165:151-60.

17. Khani M, Hosseintash M, Foroughi M, Naderian M, Khaheshi I. Assessment of the effect of off-pump coronary artery bypass (OPCAB) surgery on right ventricle function using strain and strain rate imaging. Cardiovasc Diagn Ther. 2016;6:138-43.

18. Becker M, Robbers L, Brouwer W, Beck A, Abel MD, Bondarenko O, et al. A cardiovascular magnetic resonance study on the short and long-term effects of coronary artery bypass graft surgery on the right ventricular systolic function. Madridge J Cardiol. 2017;1:14-20.

19. Lindqvist P, Holmgren A, Zhao Y, Henein MY. Effect of pericardial repair after aortic valve replacement on septal and right ventricular function. Int J Cardiol. 2012;155:388-93.

20. Hashemi N, Brodin LA, Hedman A, Samad B A, Alam M. Improved right ventricular index of myocardial performance in the assessment of right ventricular function after coronary artery bypass grafting. Interact Cardiovasc Thorac Surg. 2018;26:798-804.

21. Guinot PG, Abou-Arab O, Longrois D, Dupont H. Right ventricular systolic dysfunction and vena cava dilatation precede alteration of renal function in adult patients undergoing cardiac surgery: an observational study. Eur J Anaesthesiol. 2015;32:535-42. 
22. Desai RR, Vargas Abello LM, Klein AL, Marwick TH, Krasuski RA, Ye Y, et al. Tricuspid regurgitation and right ventricular function after mitral valve surgery with or without concomitant tricuspid valve procedure. J Thorac Cardiovasc Surg. 2013;146:1126-32.e10.

23. Schuuring MJ, Bolmers PP, Mulder BJ, de Bruin-Bon RA, Koolbergen DR, Hazekamp MG, et al. Right ventricular function declines after cardiac surgery in adult patients with congenital heart disease. Int $J$ Cardiovasc Imaging. 2012;28:755-62.

24. Keyl C, Schneider J, Beyersdorf F, Ruile P, Siepe M, Pioch K, et al. Right ventricular function after aortic valve replacement: a pilot study comparing surgical and transcatheter procedures using 3D echocardiography. Eur J Cardiothorac Surg. 2016;49:966-71.

25. Duncan AE, Sarwar S, Kateby Kashy B, Sonny A, Sale S, Alfirevic A, et al. Early left and right ventricular response to aortic valve replacement. Anesth Analg. 2017; $124: 406-18$.

26. D'Armini AM, Zanotti G, Ghio S, Magrini G, Pozzi M, Scelsi L, et al. Reverse right ventricular remodeling after pulmonary endarterectomy. J Thorac Cardiovasc Surg. 2007;133:162-8.

27. Grønlykke L, Ravn HB, Gustafsson F, Hassager C, Kjaergaard J, Nilsson JC. Right ventricular dysfunction after cardiac surgery - diagnostic options. Scand Cardiovasc J. 2017;51:114-21.

28. Coisne A, Modine T, Outteryck F, Mouton S, Pilato R, Ridon H, et al. Clinical significance of right ventricular longitudinal function parameters after aortic valve replacement. JACC Cardiovasc Imaging. 2018;11:651-2.
29. Garcia Gigorro R, Renes Carreño E, Mayordomo S, Marín H, Perez Vela JL, Corres Peiretti MA, et al. Evaluation of right ventricular function after cardiac surgery: the importance of tricuspid annular plane systolic excursion and right ventricular ejection fraction. J Thorac Cardiovasc Surg. 2016; 152:613-20.

30. Flo Forner A, Hasheminejad E, Sabate S, Ackermann MA Turton EW, Ender J. Agreement of tricuspid annular systolic excursion measurement between transthoracic and transesophageal echocardiography in the perioperative setting. Int J Cardiovasc Imaging. 2017;33: 1385-94.

31. Skinner H, Kamaruddin H, Mathew T. Tricuspid annular plane systolic excursion: comparing transthoracic to transesophageal echocardiography. J Cardiothorac Vasc Anesth. 2017:31:590-4.

Key Words: tricuspid annular plane systolic excursion (TAPSE), right ventricular (RV) function, heart surgery, coronary artery bypass surgery, perioperative echocardiography, TAPSE reduction, pericardiotomy, chest closure, transesophagealechocardiography, transthoracic, echocardiography, M-mode, anatomic M-mode, tissue Doppler velocity, pulmonary artery velocity time integral 